\title{
Editorial
}

\section{Khosrow Adeli \\ Critical role of laboratory medicine in the global response to the COVID-19 pandemic}

https://doi.org/10.1515/cclm-2020-0742

The global coronavirus disease 2019 (COVID-19) has presented major challenges for clinical laboratories, from initial diagnosis to patient monitoring and treatment. As I write this editorial, many laboratory specialists, technologists, and trainees are selflessly and tirelessly standing on the front lines of the battle against COVID-19. This is happening at hospital laboratories and at private clinical laboratories around the globe. What is becoming very clear during this crisis is that clinical laboratory operations are critical in the global fight against this unprecedented pandemic through rapid diagnosis of viral infection, serological monitoring of the affected populations, and biochemical monitoring of hospitalized patients with more severe COVID-19-induced complications. Laboratory medicine is a key driver of healthcare delivery through provision of objective data to clinicians and other healthcare workers to guide appropriate clinical decision-making. Indeed, laboratory medicine is integral to prevention, diagnosis, treatment, and management of clinical disease including such infectious disease outbreaks. It supplies health care professionals with evidence-based data necessary to provide safe, effective, and high-quality care to patients.

Unfortunately, this essential role of laboratory medicine has not been widely recognized within healthcare organizations or the public, leading to poor visibility both within the field of clinical medicine and externally with the public at large. The current worldwide pandemic has clearly started to change the public's and governmental view of the critical role that clinical laboratories play in public health and safety. It is now abundantly clear that without laboratory medicine appropriate public health measures and evidence-based care of hospitalized patients are simply not conceivable. In response to the current pandemic, the International Federation of Clinical Chemistry and Laboratory Medicine (IFCC) has established a global taskforce on COVID-19 (https:// www.ifcc.org/executive-board-and-council/eb-taskforces/ifcc-task-force-on-covid-19/) as well as an online resource called the IFCC Information Guide on COVID-19 (https://www.ifcc.org/ifcc-news/2020-03-26-ifcc-information-guide-on-covid-19/). The taskforce and the online resource are helping to provide the latest evidence and up-to-date information on population screening, diagnosis, biosafety guidelines for clinical laboratories, and biochemical monitoring of hospitalized patients with COVID-19.

This first special issue of Clinical Chemistry and Laboratory Medicine (CCLM) on COVID-19 brings together a number of important reports and reviews, presenting the latest evidence on the laboratory management of this infection and the associated clinical complications. It includes a number of reviews, opinion pieces, and original articles on the pathogenesis of COVID-19 infection, as well as its laboratory diagnosis, surveillance, and monitoring of severely affected/hospitalized patients. The issue begins with comprehensive reviews on laboratory abnormalities associated with severity and mortality of COVID-19 as well as disease progression and intravascular coagulation. Two key articles are also included from the IFCC Taskforce, one on Biosafety Measures for Preventing Infection from COVID-19 in Clinical Laboratories: IFCC Taskforce Recommendations, and the second on Molecular, Serological, and Biochemical Diagnosis and Monitoring of COVID-19. These articles not only review the current evidence but also provide practical recommendations on both laboratory biosafety as well as diagnostic/serological/biochemical markers used in infection control and monitoring. In addition, a number of thoughtful opinion articles are included that discuss the critical role of laboratory medicine in the current pandemic, potential preanalytical and analytical concerns with diagnostic/serological testing, and lessons learned from the initial COVID-19 outbreak in Wuhan, China. Importantly, a series of original studies are published that provide very useful information on the performance of serological assays, specimens used in the collection and diagnosis of COVID-19, application of routine blood tests as potential diagnostic tools, lab-based predictive markers of COVID-19-associated death, hematological and coagulation abnormalities observed, as well as urine biochemical parameters that may predict disease severity. 
This timely special issue of CCLM is a must read for all clinical laboratorians, clinicians, trainees, and other healthcare workers. It is an excellent collection of highquality reviews and original papers that together provide a wide-ranging coverage of the role that clinical laboratories play in the fight against this unparalleled and unfortunate pandemic. I recognize this is a time of immense turmoil and disruption in our lives. No one knows how long this crisis will last but one thing is certain: clinical laboratories have visibly demonstrated their vital role and value in the public health surveillance and patient care and management, and are in the front line of the global response to COVID-19. On behalf of the IFCC organization, I would like to congratulate CCLM and its editors for putting together such a valuable and timely special issue which will undoubtedly become a treasured resource for laboratory medicine specialists and other healthcare workers around the world. On behalf of the Editorial Board of CCLM, I wish to thank all the authors for their comprehensive contributions on COVID-19 and hope that the readership of the journal will find interest in their content.

Khosrow Adeli, IFCC Taskforce on COVID-19; International Federation of Clinical Chemistry and Laboratory Medicine, Milan, Italy; and Clinical Biochemistry, DPLM, The Hospital for Sick Children, University of Toronto, Toronto, ON M5P 2R6, Canada, E-mail: khosrow.adeli@sickkids.ca 